\title{
$\gamma$-Secretase inhibitors suppress the growth of leukemia and lymphoma cells
}

\author{
HANAE KOGOSHI $^{1,2}$, TAKU SATO ${ }^{1}$, TAKATOSHI KOYAMA ${ }^{2}$, NOBUO NARA ${ }^{1}$ and SHUJI TOHDA ${ }^{1}$ \\ Departments of ${ }^{1}$ Laboratory Medicine and ${ }^{2}$ Laboratory Molecular Genetics of Hematology, \\ Tokyo Medical and Dental University, Yushima 1-5-45, Bunkyo-Ku, Tokyo 113-8519, Japan
}

Received January 3, 2007; Accepted April 23, 2007

\begin{abstract}
Secretase inhibitors (GSI) suppress the growth of acute T-lymphoblastic leukemia (T-ALL) cells with NOTCH1 mutations. Recently, clinical trials of GSI for refractory T-ALL have commenced. In the present study, we examined the effects of three types of GSI; GSI-I, GSI-IX, and GSI-XII on the growth of four B-cell malignant lymphoma (B-ML) and four acute myeloid leukemia (AML) cell lines as well as four T-ALL cell lines. We found that GSI also suppressed the in vitro growth of some B-ML and AML cell lines in a dosedependent manner. Growth suppression occurred through induction of apoptosis. Expression of the HES1 gene, one of the targets of Notch signaling, was high in T-ALL cells with NOTCH1 mutations, but was low in GSI-sensitive B-ML and AML cells. GSI treatment decreased HES1 mRNA expression in T-ALL cells, while GSI increased HES1 mRNA in two GSI-sensitive B-ML and AML cell lines. In immunoblot analysis, the band for the intracellular fragment of Notch1, an active form of Notch1, was dense in T-ALL cells but was faint in GSI-sensitive B-ML and AML cells; attenuation of the band by GSI was not evident. These findings suggest that GSI may act on Notch 2, 3 or 4 protein, or some pathways other than Notch signaling in GSI-sensitive B-ML and AML cells. Namely, growth suppression by GSI may involve cell growth-related proteins, which are $\gamma$-secretase substrates. Taken together, we have shown that GSI may be useful for the treatment of hematological malignancies other than T-ALL. The mechanism behind the effects remains to be clarified. Our investigations lead to a novel molecular target therapy for chemotherapy-resistant leukemia and lymphomas.
\end{abstract}

\section{Introduction}

Notch signaling is involved in the cell growth of various hematological malignancies $(1,2)$. For example, more than

Correspondence to: Dr Shuji Tohda, Department of Laboratory Medicine, Tokyo Medical and Dental University, Yushima 1-5-45, Bunkyo-Ku, Tokyo 113-8519, Japan

E-mail: tohda.mlab@tmd.ac.jp

Key words: notch, $\gamma$-secretase inhibitor, leukemia, lymphoma, apoptosis half of acute T-lymphoblastic leukemia (T-ALL) cases have activating NOTCH1 mutations $(3,4)$. In normal hematopoietic cells, the binding of Notch ligands to Notch1 protein causes S2 and S3 cleavage of the transmembrane subunit (TM), and releases the intracellular fragment of Notch1 (ICN). The ICN then translocates to the nucleus for signal transduction. Mutations in Notch1 in T-ALL cells facilitate cleavage of the ICN and prolong the half-life, causing abnormal growth of leukemia cells. $\gamma$-Secretase inhibitors (GSI), which block Notch activation by inhibiting S3 cleavage, were shown to induce cell-cycle arrest and apoptosis in NOTCH1-mutated T-ALL cell lines $(3,5)$.

We previously investigated the roles of Notch in the proliferation of acute myeloid leukemia (AML) cells (6-8). Moreover, we recently established a novel B-cell malignant lymphoma (B-ML) cell line, the growth of which is suppressed by GSI even though it does not have NOTCH1 mutations (9). In the present study, we examined the effects of GSI on the growth of B-ML and AML cell lines as well as T-ALL cells. We also examined the molecular events in the cells treated with GSI. To our knowledge, this is the first report to evaluate the effects of GSI on B-ML and AML cells. Our study provides clues to the therapeutic use of GSI for various leukemia and lymphomas.

\section{Materials and methods}

Cells. Four T-ALL cell lines with NOTCH1 mutations (3), KOPT-K1, DND-41, PF-382, and Jurkat were donated by Dr Harashima and Dr Orita (Fujisaki Cell Center, Japan). Three Burkitt lymphoma cell lines, Ramos, Daudi and Raji, were supplied by the Japanese Cancer Research Resources Bank. Raji is positive for EBNA2 protein, which is reported to activate the Notch signaling pathway bypassing Notch protein $(10,11)$. A diffuse large B-cell lymphoma cell line, MD901, was donated by Dr T. Miki. Four AML cell lines, OCI/AML-2 and OCI/AML-3 (established at Ontario Cancer Institute, Canada), TMD7 (6) and THP-1 (supplied by the Japanese Cancer Research Resources Bank) were used.

Cell growth assay. The effects of GSI on cell growth were examined using a colorimetric (WST-1) assay. Briefly, three kinds of GSI; GSI-I (Z-LLNle-CHO), GSI-IX (DAPT), and GSI-XII (Z-IL-CHO) (Calbiochem, USA) were dissolved in dimethyl sulphoxide (DMSO). Cells $\left(1 \times 10^{4}\right.$ cells/well) were 
T-ALL

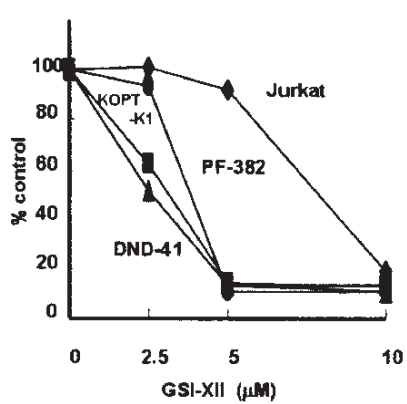

B-ML

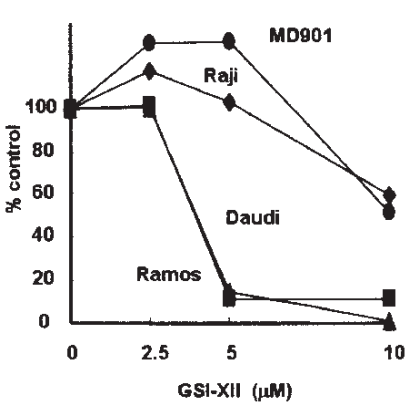

AML

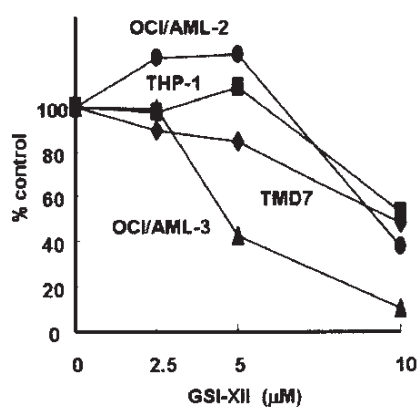

Figure 1. Dose-response curves of GSI-XII on the growth of T-ALL, B-lymphoma and AML cell lines. Cells were cultured in the presence of increasing concentrations of GSI-XII. After 2 days, growth was examined using a colorimetric (WST-1) assay. Growth is shown as a percentage of the mean OD value of the control cells cultured without GSI-XII.
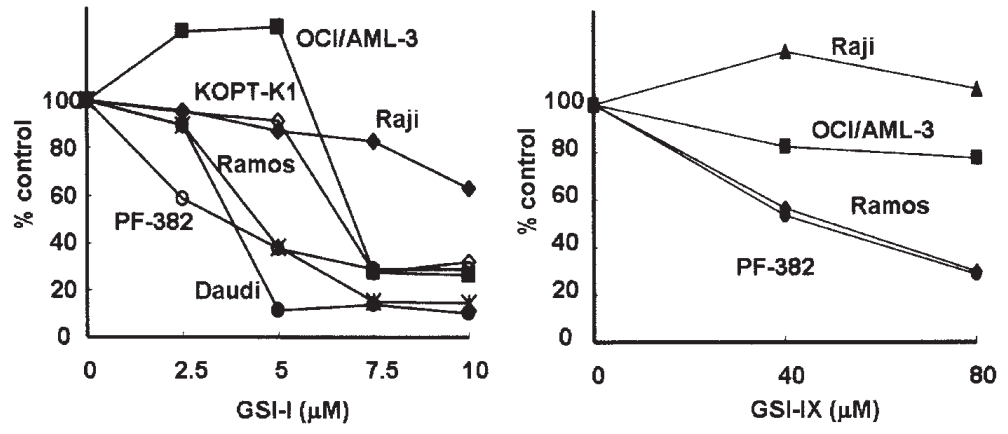

Figure 2. Dose-response curves of GSI-I and GSI-IX on the growth of representative cells. Evaluation was performed as described in Fig. 1.

then cultured in $0.1 \mathrm{ml}$ of $10 \%$ fetal calf serum-supplemented RPMI-1640 medium (Gibco BRL, USA) in the presence of increasing concentrations of GSI. After 2 days, WST-1 and 1-methoxy PMS (Dojindo Laboratories, Japan) were added at the manufacturer's recommended concentrations. The optical density (OD) was measured using an ELISA plate reader and growth was shown as a percentage of the mean OD value of control cells cultured without GSI.

Apoptosis. To evaluate apoptosis, cytospin preparations of cells cultured for $18 \mathrm{~h}$ with or without $5 \mu \mathrm{M}$ GSI-XII were stained with Wright and observed under a light microscope. The number of apoptotic cells with nuclear condensation and apoptotic bodies in 300 cells was then counted.

Quantitative RT-PCR. Cells were cultured with or without $5 \mu \mathrm{M}$ GSI-I for $6 \mathrm{~h}$. We used GSI-I because only GSI-I is soluble in acetic acid. Acetic acid did not affect HES1 mRNA expression, while DMSO affected HESI mRNA expression according to our preliminary experiments. Total RNA was extracted from the cells and then first-strand cDNA was synthesized. Quantitative PCR was performed using a FastStart DNA Master SYBR-Green I kit, LightCycler primer sets for HES1 and $\beta$-ACTIN, and LightCycler (Roche Diagnostics). HES1 mRNA expression was normalized by the $\beta$-ACTIN mRNA level, which was measured concurrently.
Immunoblotting. To examine the effects of GSI on Notch1 protein, we performed immunoblot analysis. Before and after culture with GSI-XII for $18 \mathrm{~h}$, cells were harvested and lysed. The lysates from $1 \times 10^{6}$ cells/lane were then subjected to SDS-PAGE and immunoblotted with an anti-cleaved Notch1 (Val1744) Ab (Cell Signaling Technology, USA) to selectively detect the ICN. Immunoreactive bands were detected with an ECL kit (Amersham Biosciences, USA). Subsequently, the membranes were reprobed with an antiNotch1 C-terminus Ab (Santa Cruz Biotechnology, USA). We repeated the experiments at least twice to verify the reproducibility.

\section{Results}

Growth suppression by GSI. The dose-response curves of GSI-XII on the growth of T-ALL, B-ML and AML cells are shown in Fig. 1. The growth of T-ALL cells was suppressed by GSI-XII; however, Jurkat was relatively resistant. GSI-XII also suppressed the growth of B-ML and AML cells such as Ramos, Daudi, and OCI/AML-3 in a dose-dependent manner. Treatment at a low dose of $2.5 \mu \mathrm{M}$ GSI-XII suppressed only the T-ALL cells, suggesting that T-ALL cells are more sensitive than B-ML and AML cells. We also examined the effects of GSI-I and GSI-IX and found that these two GSI suppressed growth in a similar manner to GSI-XII (Fig. 2). 
Table I. Induction of apoptosis by GSI-XII treatment.

\begin{tabular}{llccr}
\hline Type & Cell line & Control & DMSO & GSI-XII \\
\hline T-ALL & DND-41 & 0.0 & 0.0 & 20.0 \\
& PF-382 & 0.0 & 0.0 & 12.0 \\
& KOPT-K1 & 0.3 & 0.3 & 15.0 \\
& Jurkat & 0.0 & 0.3 & 2.0 \\
B-ML & Ramos & 0.0 & 0.3 & 55.0 \\
& Daudi & 0.3 & 0.3 & 15.0 \\
& Raji & 0.0 & 0.3 & 0.7 \\
AML & OCI/AML-3 & 0.0 & 0.0 & 11.0 \\
\hline
\end{tabular}

Cytospin preparations of cells cultured with $5 \mu \mathrm{M}$ GSI-XII dissolved in DMSO for $18 \mathrm{~h}$ were stained with Wright and observed under a light microscope. Values indicate the rate (\%) of apoptotic cells with nuclear condensation and apoptotic bodies in 300 cells.

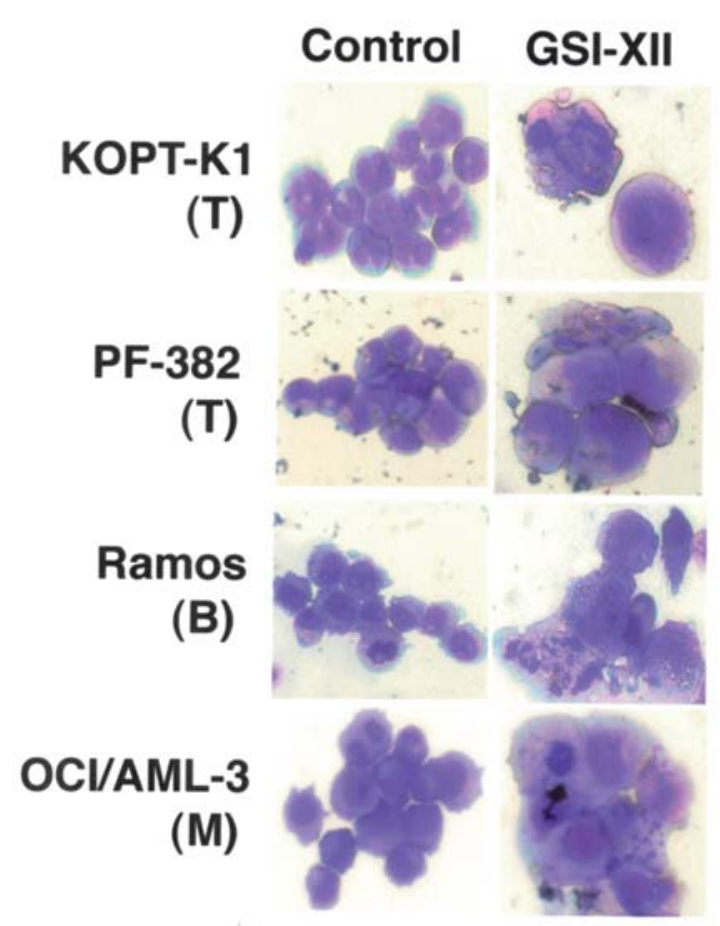

Figure 3. Effect of GSI on cell morphology. The cells were cultured with or without $5 \mu \mathrm{M}$ GSI-XII. After $18 \mathrm{~h}$, cytospin preparations of the cells were stained with Wright and observed under a light microscope (original magnification, $\mathrm{x} 400$ ). T, T-ALL; B, B-ML; and M, AML.

GSI-IX required a higher concentration to suppress growth than GSI-I and GSI-XII. In these experiments, DMSO used as a vehicle did not affect growth. We repeated these experiments in triplicate independently and obtained similar results.

Apoptosis induced by GSI. We also examined the effects of GSI-XII on cell morphology. As shown in Fig. 3, apoptotic cells with nuclear condensation and apoptotic bodies were observed in cells showing strongly suppressed growth as a result of GSI. Apoptosis was induced in B-ML and AML as well as in T-ALL cells (Table I).

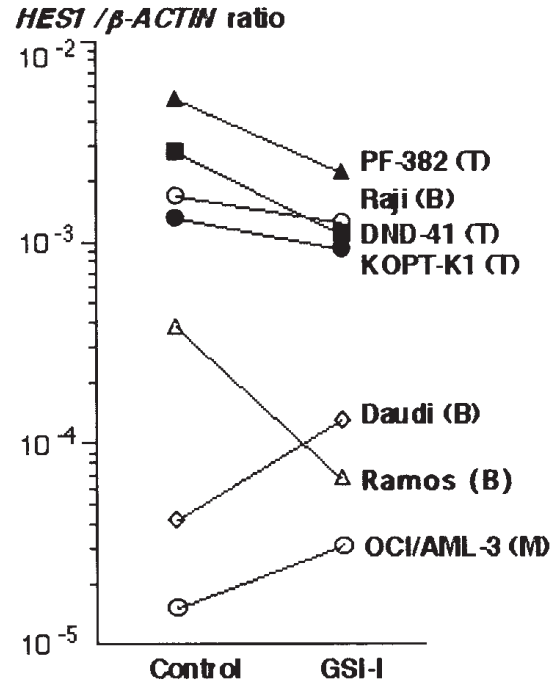

Figure 4. HES1/ $\beta$-ACTIN mRNA ratios in cells cultured with GSI-I. RNA was extracted from cells cultured with $5 \mu \mathrm{M}$ GSI-I dissolved in acetic acid or with acetic acid only (control) for $6 \mathrm{~h}$. Quantitative RT-PCR was performed. The HES1 mRNA expression level was normalized by the $\beta$ ACTIN mRNA expression level.

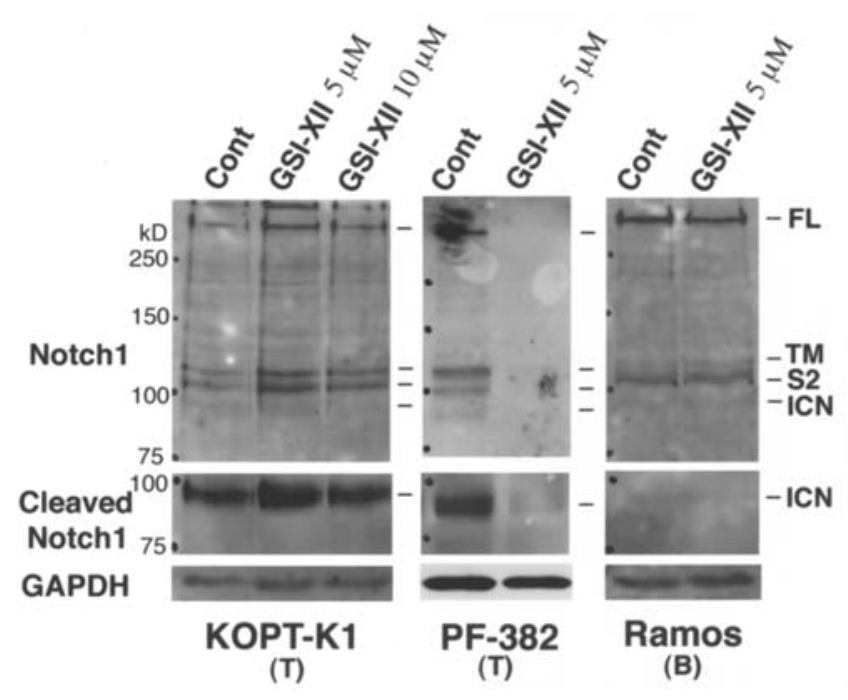

Figure 5. Expression of Notch1 proteins in cells exposed to GSI-XII. Cells cultured with GSI-XII for $18 \mathrm{~h}$ were harvested. The lysates $\left(10^{6}\right.$ cells/lane $)$ were then subjected to SDS-PAGE and immunoblotted with anti-Notch1 C-terminus Ab and anti-cleaved Notch1 (Val1744) Ab. FL, full-length; TM, transmembrane subunit; S2, S2 cleavage fragment; and ICN, intracellular fragment of Notch1.

Effects of GSI on HES1 mRNA expression. As shown in Fig. 4, HES1 mRNA expression was high in T-ALL cells and low in AML and B-ML cells, except for that in Raji. GSI treatment decreased HESI mRNA except for that in Daudi and OCI/ AML-3 cells.

Effects of GSI on the cleavage of ICN. Fig. 5 shows immunoblots with the anti-Notch1 C-terminus $\mathrm{Ab}$ and anti-cleaved Notch1 Ab. In the upper panel, a 300-kDa band represents the full-length (FL) Notch protein, while a $120-\mathrm{kDa}$ band 
represents the transmembrane subunit (TM). A 110-kDa band seems to be an S2 cleavage fragment (S2), according to its weight. In the middle panel, ICN bands of KOPT-K1 and PF-382 were observed at $290-97$ and 85-95 kDa, respectively. The ICN band of Ramos (93 kDa) was so faint that it was not clearly recognized in this figure. Treatment with KOPT-K1 and GSI-XII up to $10 \mu \mathrm{M}$ did not affect the FL, TM, S2 and ICN bands, while treatment with $20 \mu \mathrm{M}$ GSI-XII decreased the cell viability; therefore, the intact protein could not be extracted from these cells (data not shown). DND-41 showed similar results to KOPT-K1 (data not shown). In PF-382, GSI treatment suppressed the expression of the Notch protein rather than release of the ICN. In Ramos, it was difficult to evaluate the change in the ICN band by GSI as the band was so weak. OCI/AML-3 also showed a faint ICN band, which was not significantly affected by GSI treatment (data not shown).

\section{Discussion}

In this study, we found that GSI suppressed the growth of not only T-ALL cells but also some types of B-ML and AML cells through induction of apoptosis at relatively low concentrations. It has previously been reported that the growth of a Kaposi's sarcoma cell line was suppressed by $10 \mu \mathrm{M}$ GSI-I (12) and that Ras-transformed fibroblasts were suppressed by $25 \mu \mathrm{M}$ GSI-XII (13). The T-ALL cells examined in this study showed high HES1 mRNA expression and dense ICN bands, indicating that Notch 1 was highly activated. The GSIsensitive B-ML and AML cells such as Ramos, Daudi and OCI/AML-3 showed low HES1 mRNA expression and faint ICN bands, indicating that Notch1 activity was low. On the other hand, Jurkat was GSI-resistant even though it has activating NOTCH1 mutations. Thus, high Notch1 activity is not a necessary and sole cause of sensitivity to GSI.

As GSI is thought to block the S3 cleavage, we first hypothesized that GSI treatment would attenuate the ICN band and increase the S2 cleavage fragment in immunoblots. Recently, Malecki et al reported that in cells with mutated NOTCH1-expression vectors, S2 cleavage products were accumulated by GSI treatment (5). We previously reported that GSI treatment attenuated the ICN band of a GSI-sensitive T-ALL cell line, ALL-SIL (9). On the other hand, in a GSIsensitive B-ML cell line, TMD8, its faint ICN band was not evidently affected by GSI (9). The present study also showed that, in the GSI-sensitive B-ML and AML cells, GSI treatment did not decrease HES1 mRNA expression and did not clearly change the ICN band. These findings suggest that GSI may act on Notch 2, 3, or 4 protein, or pathways other than Notch signaling. Many proteins such as cadherin and CD44 are known to be $\gamma$-secretase substrates (14), and growth suppression by GSI may involve these cell growth-related proteins.

Notably, in a GSI-sensitive T-ALL cell line, KOPT-K1, the ICN band was not evidently affected by 5-10 $\mu \mathrm{M}$ GSI even though this concentration of GSI suppressed growth. In PF-382, GSI decreased the expression of Notch1 protein itself rather than the $\mathrm{S} 3$ cleavage. These findings suggest that GSI may not simply inhibit the release of ICN, but rather act on alternative pathways even in T-ALL cells with NOTCH1 mutations.
As for the mechanisms of Notch activation in AML cells without NOTCH1 mutations, we proposed the possibility of cell-autonomous Notch activation based on the finding that AML cells not only express Notch receptors but also the Notch ligand protein (6). In Raji, EBNA2 binds to RBP-Jk and activates Notch signaling while bypassing the Notch protein $(13,14)$. Therefore, GSI do not suppress Notch-HES signaling. This is one possible reason why Raji cells are GSI resistant. In other B-ML cells, the mechanisms of Notch activation and its roles are still unknown (2).

Clinical trials with GSI for refractory T-ALL have recently begun in the U.S. As shown here, GSI may also be useful for the treatment of other hematological malignancies. For this purpose, we must clarify the mechanism(s) behind such effects. Our on-going investigations lead to a novel molecular target therapy for chemotherapy-resistant leukemia and lymphomas.

\section{References}

1. Nickoloff BJ, Osborne BA and Miele L: Notch signaling as a therapeutic target in cancer: a new approach to the development of cell fate modifying agents. Oncogene 22: 6598-6608, 2003.

2. Leong KG and Karsan A: Recent insights into the role of Notch signaling in tumorigenesis. Blood 107: 2223-2233, 2006.

3. Weng AP, Ferrando AA, Lee W, Morris JP IV, Silverman LB, Sanchez-Irizarry C, Blacklow SC, Look AT and Aster JC: Activating mutations of NOTCH1 in human T cell acute lymphoblastic leukemia. Science 306: 269-271, 2004.

4. Lee SY, Kumano K, Masuda S, Hangaishi A, Takita J, Nakazaki K, Kurokawa M, Hayashi Y, Ogawa S and Chiba S: Mutations of the Notch1 gene in T-cell acute lymphoblastic leukemia: analysis in adults and children. Leukemia 19: 1841-1843, 2005.

5. Malecki MJ, Sanchez-Irizarry C, Mitchell JL, Histen G, Xu ML, Aster JC and Blacklow SC: Leukemia-associated mutations within the NOTCH1 heterodimerization domain fall into at least two distinct mechanistic classes. Mol Cell Biol 26: 4642-4651, 2006.

6. Tohda S, Sakano S, Ohsawa M, Murakami N and Nara N: A novel cell line derived from de novo acute myeloblastic leukaemia with trilineage myelodysplasia which proliferates in response to a Notch ligand, Delta-1 protein. Br J Haematol 117: 373-378, 2002.

7. Kogoshi H, Tohda S, Fu L and Nara N: Effect of Notch ligands on in vitro sensitivity to chemotherapeutic drugs in leukemia and lymphoma cells. Oncol Rep 14: 723-726, 2005.

8. Tohda S, Kogoshi H, Murakami N, Sakano S and Nara N: Diverse effects of the Notch ligands Jagged1 and Delta1 on the growth and differentiation of primary acute myeloblastic leukemia cells. Exp Hematol 33: 558-563, 2005.

9. Tohda S, Sato T, Kogoshi H, Fu L, Sakano S and Nara N: Establishment of a novel B-cell lymphoma cell line with suppressed growth by gamma-secretase inhibitors. Leuk Res 30: 1385-1390, 2006

10. Chiaramonte R, Calzavara E, Balordi F, Sabbadini M, Capello D, Gaidano G, Serra A, Comi P and Sherbet GV: Differential regulation of Notch signal transduction in leukemia and lymphoma cells in culture. J Cell Biochem 88: 569-577, 2003.

11. Hofelmayr H, Strobl LJ, Marschall G, Bornkamm GW and Zimber-Strobl U: Activated Notch1 can transiently substitute for EBNA2 in the maintenance of proliferation of LMP1expressing immortalized B cells. J Virol 75: 2033-2040, 2001.

12. Curry CL, Reed LL, Golde TE, Miele L, Nickoloff BJ and Foreman KE: Gamma secretase inhibitor blocks Notch activation and induces apoptosis in Kaposi's sarcoma tumor cells. Oncogene 24: 6333-6344, 2005.

13. Weijzen S, Rizzo P, Braid M, Vaishnav R, Jonkheer SM, Zolbin A, Osborne BA, Gottipati S, Aster JC, Hahn WC, Rudolf M, Siziopikou K, Kast WM and Miele L: Activation of Notch-1 signaling maintains the neoplastic phenotype in human Ras-transformed cells. Nat Med 8: 979-986, 2002.

14. Kopan R and Ilagan MXG: $\gamma$-Secretase: proteasome of the membrane? Nat Rev Mol Cell Biol 5: 499-504, 2004. 